\title{
INVESTIGACIÓN
}

\section{Confiabilidad de la Escala Apoyo Social en Redes Sociales para el manejo del peso corporal}

\author{
Guevara-Valtier, Milton Carlos"i*; González-De la Cruz, Patricia²; Chávez-Herrara, Alina Edith³; \\ Cárdenas-Villarreal, Velia Margarita4; Gutiérrez-Valverde, Juana Mercedes ${ }^{5}$
}

\begin{abstract}
RESUMEN
Introducción: La adolescencia es considerada una etapa crítica en la que se podrían adoptar estilos de vida poco saludables que contribuyen al desarrollo de enfermedades en la edad adulta, este panorama aumenta las posibilidades para que los adolescentes se interesen y preocupen por el manejo del peso corporal, en este sentido los consejos, aliento y recomendaciones sobre una alimentación saludable son de importancia para lograr resultados favorables, lo anterior es un tipo de apoyo social que puede encontrarse en ambientes virtuales o redes sociales. Objetivo: Diseñar y valorar un primer análisis de confiabilidad de una escala corta para valorar el apoyo social percibido por adolescentes en redes sociales para el manejo del peso corporal basado en una alimentación saludable (Fase I). Metodología: Diseño descriptivo polietápico, se diseñó y valoró la confiabilidad de la escala apoyo social percibido por adolescentes en redes sociales para el manejo del peso corporal basado en una alimentación saludable, se aplicó a 32 adolescentes. Resultado: El alfa de Cronbach que demostró el cuestionario fue 0.85. Conclusión: El cuestionario demostró una confiabilidad aceptable.
\end{abstract}

Palabras clave: Reproducibilidad de los resultados, apoyo social, internet, dieta saludable (DeCS, BIREME).

1.Doctorado en Educación. Universidad Autónoma de Nuevo León. E-mail: carlos_valtier7@hotmail.com

2.Maestría en Ciencias de Enfermería. Universidad Veracruzana.

3.Maestría en Ciencias de Enfermería. Universidad Autónoma de Campeche.

4.Doctora en Cultura del Cuidado de Enfermería. Universidad Autónoma de Nuevo León.

5.Doctora en Ciencias de Enfermería. Universidad Autónoma de Nuevo León.

*Autor para correspondencia

\section{Cómo citar este artículo}

Guevara-Valtier MC, González-De la Cruz P, Chávez-Herrara AE; Cárdenas-Villarreal VM, Gutiérrez-Valverde JM. Confiabilidad de la Escala Apoyo Social en Redes Sociales para el manejo del peso corporal. Sanus. 2018;3(6): 8-19. [Acceso _____ ]; Disponible en: $\overline{\text { mes }} \overline{\text { día año }}$ URL 


\section{INTRODUCCIÓN}

La adolescencia es considerada una etapa especial de la vida, se atribuye a la presencia de cambios biológicos, psicológicos, intelectuales y sociales que pueden afectar positiva o negativamente la salud de este grupo poblacional $\left({ }^{(1,2)}\right.$, en este sentido la adopción de estilos de vida poco o nada saludables como el sedentarismo y el consumo de alimentos altos en grasa son factores que en un futuro pueden predecir el desarrollo de enfermedades crónicas degenerativas como hipertensión arterial o diabetes mellitus tipo II. De acuerdo a estudios realizados los resultados indican datos alarmantes, en México 4.2\% de adolescentes tenía un Estilo de Vida (EV) no saludable y $63 \%$ del grupo poblacional tenía sobrepeso u obesidad y además $79.5 \%$ eran inactivos físicamente. En otro estudio realizado en Chile se encontraron 3.6\% de una muestra analizada tenía sobrepeso/obesidad (3,4). En España se encontró en una muestra representativa de universitarios un patrón excesivo de consumo de carnes y derivados, incluyendo grasas y dulces (>120\%). Sin embargo, el consumo de aceites como el de oliva y girasol, cereales especialmente los integrales y papa, frutos secos, legumbres, frutas y verduras, presentan un consumo insuficiente $(<80 \%)$, el grupo de alimentos de huevo, pescado y lácteos es consumido en exceso por la mitad de los estudiantes y de forma insuficiente hasta por un cuarto de ellos ${ }^{(5)}$. En Colombia se evidenció que de un $65 \%$ de menores de edad, entre estos adolescentes son inactivos físicamente y solo $7.5 \%$ no tiene un buen consumo de frutas y verduras ${ }^{(6)}$.

Aunado a lo anterior estudios recientes confirman que los adolescentes perciben equivocadamente su peso real en comparación con la auto percepción de su figura corporal, es decir poseen una visión inadecuada considerando el Índice de Masa Corporal real, situación que incrementa la vulnerabilidad de desarrollar enfermedades sobre todo de tipo crónico degenerativas ${ }^{(7,8)}$. Al respecto en Colombia se realizó un estudio en una muestra de estudiantes, se encontró una percepción de peso corporal baja en 48 (15.0\%); adecuada en 228 (71.0\%); y alta en 45 (14.0\%). Los IMC se observaron entre 14.5 y 29.8, media $20.5(D E=3.0)^{(9)}$.

El panorama descrito plantea la necesidad de considerar los recursos con los que los adolescentes tienen contacto y cuentan para afrontar los cambios ${ }^{(10)}$. Un recurso que es potencialmente útil en la etapa de la adolescencia es el apoyo que estos pueden recibir u obtener en una red social (11). El Apoyo Social (AS) puede extenderse de acuerdo a las posibilidades de los proveedores y receptores como tener contacto con personas que experimenten salud o enfermedad con el fin de buscar e intercambiar experiencias. En este sentido una forma de evaluar el AS que los adolescentes reciben por medio de redes sociales consiste en el uso de cuestionarios en versiones reducidas con propiedades psicométricas apropiadas que disminuyan los efectos adversos durante su aplicación como fatiga y falta de motivación para su cumplimentación ${ }^{(12)}$
En este sentido se analizaron diferentes cuestionarios que evalúan AS, todos superiores en reactivos al test propuesto en este estudio además que no evalúan el AS percibido en redes sociales como facebook, twitter, instagram u otras; entre los cuestionarios valorados fueron el de afrontamiento, apoyo social y conducta alimentaria en personas con diabetes e hipertensión con 23 preguntas, este cuestionario presenta datos de validez y confiabilidad adecuados $(\mathrm{B}=1755, p<0.01, \mathrm{KMO}=0.788 \text { y } \alpha=0.547 \text { a } 0.915)^{(13)}$, el Social Support Questionaire de Evaluación de Redes de Apoyo Social con un total de 27 reactivos $(\alpha=0.940)$, este cuestionario ha demostrado correlaciones adecuadas con ítems de otros cuestionarios que evalúan AS $(p<0.03){ }^{(14)}$ y el cuestionario Medical Outcomes Study (MOS) conformado por 20 ítems con 5 opciones de respuesta (de 1 a 5); el primero se utiliza para indagar el apoyo estructural y el resto de tipo funcional, dividido en 4 sub escalas denominadas de apoyo emocional, instrumental, interacción social positiva y afectivo, este cuestionario ha presentado alfa de Cronbach de 0.97 para su puntuación total (15).

Sin embargo, fue a partir de la escala para evaluar el AS a través de redes sociales (17) mismo que a su vez se desarrolló a partir del MOS del que se tomó la base de 5 preguntas adaptándose frases relacionadas con el AS para el manejo del peso corporal considerando una alimentación saludable, por ejemplo: Cuándo pides algún tipo de apoyo a tus amigos (as) como consejos de alimentación saludable para manejar tu peso corporal ¿Te responden (como: hablarte, ir a verte, etc.)?

El objetivo de este estudio fue diseñar y valorar un primer análisis de confiabilidad de una escala corta para valorar el Apoyo social percibido por adolescentes en Redes Sociales para el manejo del Peso Corporal basado en una Alimentación Saludable (Fase I).

\section{METODOLOGÍA}

El diseño del estudio fue descriptivo polietápico, ya que se describió una situación que ocurre de manera natural y debido a que se reporta la fase de diseño y las propiedades psicométricas (Alpha de Cronbach) de la Escala de Apoyo Social Para una Alimentación Saludable (EASPAS) ${ }^{(16)}$. Se realizó una prueba piloto a una muestra de 32 adolescentes inscritos en una universidad pública. El cuestionario fue respondido por quienes aceptaron tener acceso a una cuenta de Facebook, Twiter, Instagram u otra.

EI EASPAS se diseñó utilizando como fuente de partida el cuestionario original denominado titulado Escala de Apoyo Social en Redes Sociales (ASRS), la escala se compone de 15 ítems, el ítem número 1 hace referencia al tamaño de la red social y los 14 items restantes hacen referencia a cuatro dimensiones del apoyo social funcional: emocional/informacional, instrumental, interacción social positiva y apoyo 
afectivo. La escala tiene un formato de respuesta tipo Likert ( $4=$ mucho, algo, más o menos, casi nada y $0=$ nada). Todos los ítems puntúan de forma positiva. Las pruebas psicométricas del instrumento indican una adecuada consistencia interna de $(a=.94)$ según el alfa de Cronbach. Asimismo, la validez original muestra una varianza explicada del 84.9 (17).

La modificación de la escala que dio por resultado la EASPAS consistió en adaptar el contenido de cada pregunta enfocándola en el AS que las personas jóvenes pueden percibir a través de redes sociales para el manejo de su peso corporal considerando la realización de alimentación saludable.

Por lo que la adaptación y análisis del mismo se dividió en 2 fases, en primer orden se analizaron los ítems relacionados con la "alimentación saludable" y en un análisis futuro se llevará a cabo el análisis de los ítems relacionados con el ASRS para la realización de actividad física saludable para el manejo del peso corporal. Los ítems que evalúan el ASRS relacionados con el manejo del peso con base a una alimentación saludable son 5 reactivos y tiene una escala de respuestas tipo Likert que va de 5 a 1 donde 5 corresponde a mucho y 1 nada de apoyo social, la sumatoria de los puntos refleja totales mínimos y máximos que van de 5 a 25 lo que indica que entre mayor sea el puntaje obtenido mayor es la percepción de apoyo social en redes sociales para manejar el peso corporal con base a una alimentación saludable. La EASPAS es una escala sencilla de contestar que facilita su aplicación.

Para la realización de esta prueba piloto en primera instancia el protocolo fue sometido al Comité de Ética de la institución auspiciadora se obtuvo autorización cuyo registro es FAEN-L-1350, se respetó el derecho a participar voluntariamente, privacidad, anonimato y retiro bajo decisión de los participantes aspectos señalados en la Ley General de Salud y a los principios éticos de la declaración de Helsinki ${ }^{(18,19) .}$

Se empleó estadística descriptiva y el análisis de confiabilidad se realizó mediante el coeficiente alfa de cronbach, los resultados de este análisis se contrastaron contra valores de entre 0.70 a 1.00, si el resultado se ubicaba entre estos se consideró aceptable ${ }^{(17)}$, además de lo anterior se obtuvieron resultados descriptivos mediante el empleo de medidas de tendencia central, frecuencias y porcentajes. Los datos se procesaron con el paquete estadístico SPSS 23.

\section{RESULTADOS}

La media de edad de los adolescentes fue de 17 años $(\mathrm{DE}=1.5), 60 \%$ eran mujeres. En la tabla 1, se describe el porcentaje de participantes por categoría ítem/respues-

Tabla 1. Porcentaje de participantes por categoría de ítem/respuesta

\begin{tabular}{|c|c|c|c|c|c|c|c|c|c|c|}
\hline Ítem & Much & $10(5)$ & $\begin{array}{l}\text { Al } \\
\text { ( }\end{array}$ & & $\begin{array}{r}\text { Má } \\
\text { men }\end{array}$ & $\begin{array}{l}50 \\
5 \text { (3) }\end{array}$ & Casi & $\begin{array}{l}\text { nada } \\
\text { ?) }\end{array}$ & & \\
\hline & $f$ & $\%$ & $f$ & $\%$ & $f$ & $\%$ & $f$ & $\%$ & $f$ & $\%$ \\
\hline $\begin{array}{l}\text { 1. ¿Tus amigos te ayudan a resolver problemas } \\
\text { sobre una alimentación saludable para manejar } \\
\text { tu peso corporal? }\end{array}$ & 1 & 3.1 & 3 & 9.4 & 8 & 25.0 & 5 & 15.6 & 15 & 46.9 \\
\hline $\begin{array}{l}\text { 2. ¿Recibes consejos de tus amigos (as) sobre cómo } \\
\text { llevar una alimentación saludable para manejar } \\
\text { tu peso corporal? }\end{array}$ & 1 & 3.1 & 4 & 12.5 & 7 & 21.9 & 10 & 31.3 & 10 & 31.3 \\
\hline $\begin{array}{l}\text { 3. ¿Cuentas con tus amigos (as); Te dan ánimos } \\
\text { para llevar una alimentación saludable para } \\
\text { manejar tu peso corporal? }\end{array}$ & 7 & 21.9 & 9 & 28.1 & 9 & 28.1 & 4 & 12.5 & 3 & 9.4 \\
\hline $\begin{array}{l}\text { 4. ¿Les platicas a tus amigos (as) sobre la } \\
\text { alimentación que llevas para manejar tu peso } \\
\text { corporal? }\end{array}$ & 6 & 18.8 & 8 & 25.0 & 5 & 15.6 & 9 & 28.1 & 4 & 12.5 \\
\hline $\begin{array}{l}\text { 5. Cuándo pides algún tipo de apoyo a tus amigos } \\
\text { (as) como consejos de alimentación saludable } \\
\text { para manejar tu peso corporal ¿Te responden } \\
\text { (hablarte, ir a verte, etc.)? }\end{array}$ & 7 & 721.9 & 4 & 12.5 & 12 & 37.5 & 6 & 18.8 & 3 & 9.4 \\
\hline
\end{tabular}


ta, se encontró que con respecto a la percepción del apoyo recibido por los amigos para resolver problemas sobre una alimentación saludable para manejar el peso corporal, $6.9 \%$ señaló no percibir nada de apoyo social, 25.0\% más o menos reciben apoyo social (pregunta 1), con respecto a la pregunta 3 en la que se indaga si los amigos de la red social Facebook, Twiter, Instagram u otra, proporcionan ánimos para llevar una alimentación saludable para manejar el peso corporal, $28.1 \%$ recibe algo de apoyo y con un mismo porcentaje otro grupo percibe más o menos apoyo social.

Tras ejecutar el coeficiente confiabilidad se encontró un resultado aceptable de $0.85^{(17)}$ en la tabla 2 se aprecia que cada pregunta seleccionada contribuyó de forma importante a la consistencia interna global del cuestionario en su versión corta (EASPA). En la tabla 3 se aprecia la versión final del cuestionario propuesto.

\section{DISCUSIÓN Y CONCLUSIONES}

Los resultados del presente estudio indican una consistencia interna aceptable. La versión reducida para identificar el apoyo social que los adolescentes perciben con respecto a recomendaciones, $u$ otros recursos especialmente sobre llevar una alimentación saludable son consistentes con la escala a partir de la cual se modificó y diseño (17).

Existe la posibilidad de que los resultados consistentes con la versión original a partir de la cual se diseñó la versión corta del cuestionario puedan deberse a situaciones que motivan a los estudiantes a hacer uso positivo de las redes sociales; en este sentido por el hecho de tratarse de adolescentes que cursan los primeros años de una carrera profesional y del área de la salud este sea un factor que con- tribuya a la homogeneidad de los hallazgos identificados, en conjunto con lo anterior existe la posibilidad de que por tratarse de jóvenes con necesidades muy específicas, como tener dudas sobre su salud, en especial para el manejo del peso corporal a través de seguir un estilo de vida basado en una alimentación las relaciones abiertas y más grandes en las redes sociales sean un factor que contribuya a los alfas de Cronbach en comparación con el cuestionario original, es decir, 0.85 versus 0.94

Otras investigaciones han analizado anteriormente la relación que existen entre el apoyo social percibido y las actitudes y usos de las redes sociales online en usuarios de diferentes entidades diagnósticas. Asimismo, se ha estudiado el apoyo social en estos grupos en función de la edad o del género, variables que se tomaron en cuenta en este estudio $(20,21)$

En la actualidad el uso frecuente de las tecnologías de la información y la comunicación generan posibilidades de reemplazar, aunque con ciertos aspectos que vuelven peculiar la comunicación mediatizada por un equipo tecnológico; sin embargo, no existe duda que pueden ser utilizadas para obtener recursos o cubrir necesidades de diferente índole, por ejemplo la posibilidad de pedir apoyo sobre dudas o experiencias de pares relacionadas con la ejecución de un tratamiento exitoso o la prevención de conductas que potencian el riesgo para el desarrollo de enfermedades propias de la adolescencia (22).

Se concluye que el cuestionario en su versión reducida ofrece buena consistencia interna; se deben generar estudios con muestras superiores para valorar la estabilidad de los resultados y contribuir a la línea del conocimiento del ASRS.

Tabla 2. Coeficientes de confiabilidad (alfa de Cronbach) por ítem del cuestionario EASPA (Versión corta)

\begin{tabular}{llc}
\multicolumn{1}{c}{ Ítem } & Alfa de Cronbach \\
\hline 1. & $\begin{array}{l}\text { ¿Tus amigos te ayudan a resolver problemas sobre una alimentación } \\
\text { saludable para manejar tu peso corporal? }\end{array}$ & 0.83 \\
\hline 2. & $\begin{array}{l}\text { ¿Recibes consejos de tus amigos (as) sobre cómo llevar una alimentación } \\
\text { saludable para manejar tu peso corporal? }\end{array}$ & 0.82 \\
\hline 3. & $\begin{array}{l}\text { ¿Cuentas con tus amigos (as); Te dan ánimos para llevar una alimentación } \\
\text { saludable para manejar tu peso corporal? }\end{array}$ & 0.86 \\
\hline 4. & ¿Les platicas a tus amigos (as) sobre la alimentación que llevas para manejar \\
tu peso corporal? & 0.81 \\
\hline 5. & $\begin{array}{l}\text { Cuándo pides algún tipo de apoyo a tus amigos (as) como consejos de } \\
\text { alimentación saludable para manejar tu peso corporal ¿Te responden } \\
\text { (hablarte, ir a verte, etc.)? }\end{array}$ \\
\hline
\end{tabular}


Tabla 3. Versión final del Cuestionario EASPA (Versión corta)

\begin{tabular}{|c|c|c|c|c|c|}
\hline Ítem & Mucho & Algo & $\begin{array}{l}\text { Más o } \\
\text { menos }\end{array}$ & $\begin{array}{l}\text { Casi } \\
\text { nada }\end{array}$ & Nada \\
\hline $\begin{array}{l}\text { 1. ¿Tus amigos te ayudan a resolver problemas } \\
\text { sobre una alimentación saludable para manejar } \\
\text { tu peso corporal? }\end{array}$ & 5 & 4 & 3 & 2 & 1 \\
\hline $\begin{array}{l}\text { 2. ¿Recibes consejos de tus amigos (as) sobre cómo } \\
\text { llevar una alimentación saludable para manejar tu } \\
\text { peso corporal? }\end{array}$ & 5 & 4 & 3 & 2 & 1 \\
\hline $\begin{array}{l}\text { 3. ¿Cuentas con tus amigos (as); Te dan ánimos para } \\
\text { llevar una alimentación saludable para manejar tu } \\
\text { peso corporal? }\end{array}$ & 5 & 4 & 3 & 2 & 1 \\
\hline $\begin{array}{l}\text { 4. ¿Les platicas a tus amigos (as) sobre la alimentación } \\
\text { que llevas para manejar tu peso corporal? }\end{array}$ & 5 & 4 & 3 & 2 & 1 \\
\hline $\begin{array}{l}\text { 5. Cuándo pides algún tipo de apoyo a tus amigos } \\
\text { (as) como consejos de alimentación saludable } \\
\text { para manejar tu peso corporal ¿Te responden } \\
\text { (hablarte, ir a verte, etc.)? }\end{array}$ & 5 & 4 & 3 & 2 & 1 \\
\hline
\end{tabular}

Fuente: EASPA

$\boldsymbol{n}=32$

\section{REFERENCIAS BIBLIOGRÁFICAS}

1. Vélez Álvarez C, Betancurth Loaiza DP. Estilos de vida en adolescentes escolarizados de un municipio colombiano. Rev Cubana Pediatr [Internet]. 2015 [Citado 8 diciembre 2017];87(4):440-48. Disponible en: http://scielo.sld.cu/scielo. php?script $=$ sci_arttext \&pid $=$ S0034-75312015000400006\& $\mid-$ $\mathrm{ng}=\mathrm{es}$

2. Cingolani JM. Aplicación y evaluación de un programa de intervención psicosocial para promover el desarrollo saludable en adolescentes: proyecto de investigación. Anuario de Proyectos e Informes de Investigación de Becarios de Investigación [Internet]. 2009 [Citado 10 Diciembre 2017];6:2047. Disponible en: http://www.mdp.edu.ar/psicologia/psico/ investigacion/filesinves/anuario2009.pdf

3. Solórzano MC, Arellano A, Cruz B, Flores J, Mejía A, Nava $\mathrm{M}$, et al. Estilos de vida en adolescentes de nivel medio superior de una comunidad semiurbana. Eureka [Internet]. 2013 [Citado10 Diciembre 2017];10(1):77-85. Disponible en: http://pepsic.bvsalud.org/scielo.php?script=sci_arttext\&pi$d=$ S2220-90262013000100009

4. Espinoza OL, Rodríguez RF, Gálvez CJ, MacMillan KN. Hábitos de alimentación y actividad física en estudiantes universitarios. Rev. chil. Nutr [Internet]. 2011 [Citado 11 Diciembre 2017];38(4):458-65. Disponible en: https://scielo.conicyt.cl/scielo.php?script=sci_abstract\&pi-
$d=S 0717-75182011000400009 \& \operatorname{lng}=$ es \&nrm=iso

5. Ruiz Moreno E, Del Pozo de la Calle S, Valero Gaspar T, Ávila Torres JM, Varela-Moreias G. Estudio de hábitos alimentarios y estilos de vida de los universitarios españoles. Patrón de consumo de bebidas fermentadas [Internet]. España: Fundación Española de la Nutrición;2014 [Citado 11 Diciembre 2017]. 21 p. Disponible en: https://www.fen.org.es/ storage/app/media/imgPublicaciones/30092014131915.pdf

6. Campo-Ternera L, Herazo-Beltrán Y, García-Puello F, Suarez-Villa M, Méndez O, Vásquez-De la Hoz F. Estilos de vida saludables de niños y niñas y adolescentes. Salud Uninorte [Internet]. 2017 [Citado 20 Diciembre 2017]; 33(3): 419-28. Disponible en: http://www.scielo.org.co/pdf/sun/ v33n3/2011-7531-sun-33-03-00419.pdf

7. Eddy Ives LS, Moral Peláez I, Brotons Cuixart C, De Frutos Gallego E, Calvo Terrades M, Curell Aguila N. Hábitos de estilo de vida en adolescentes con sobrepeso y obesidad: Estudio Obescat. Rev Pediatr Aten Primaria [Internet]. 2012 [Citado 2 Enero 2018];14(54):127-37. Disponible en: http:// dx.doi.org/10.4321/S1139-76322012000300005

8. Hidalgo Rasmussen CA, Hidalgo-San Martín A. Percepción del peso corporal, comportamiento de control de peso y calidad de vida en adolescentes mexicanos estudiantes de secundaria. Rev Mex de Trastor Alimen [Internet]. 2011 [Citado 5 Enero 2018];2(2): 77-81. Disponible en: http://www.scielo.org.mx/scielo.php?script=sci_arttext\&pi$d=$ S2007-15232011000200003 
9. Herazo E, Villamil-Vargaz M. Concordancia entre la autopercepción de peso y el índice de masa corporal en adolescentes. Rev. salud pública [Internet]. 2012 [Citado 20 Enero 2018];14(6):1043-8. Disponible en : http://www.scielo.org.co/ pdf/rsap/v14n6/v14n6a14.pdf

10. Musaiger AO, Zaal AA, D'Souza R. Body weight perception among adolescents in Dubai, United Arab Emirates. Nutr Hosp [Internet]. 2012 [Citado 20 Enero 2018];27(6):. Disponible en: $h t t p: / / s c i e l o . i s c i i i . e s / s c i e l o . p h p ? s c r i p t=s c i \_a r t t e x-$ t\&pid $=$ S0212-16112012000600024

11. Musitu G, Cava MJ. El rol del apoyo social en el ajuste de los adolescentes. Intervención Psicosocial [Internet]. 2003 [Citado 21 Enero 2018];12(2):179-92. Disponible en: https:// www.uv.es/lisis/mjesus/8cava.pdf

12. Balluerka N, Gorostiaga A. Elaboración de Versiones Reducidas de Instrumentos de Medida: Una Perspectiva Práctica. Psychosocial Intervention [Internet]. 2012 [Citado 22 Enero 2018];21(1):103-10. Disponible en: https://www.sciencedirect.com/science/article/pii/S1132055912700686

13. Franco P, Urtubey X, Gallardo AM. Estudio de propiedades psicométricas de un cuestionario de afrontamiento, apoyo social y conducta alimentaria en personas con diabetes e hipertensión. Salud \& Sociedad [Internet]. 2017 [Citado 22 Enero 2018]; 2(8):156-70. Disponible en: https://www. researchgate.net/publication/320312267_Estudio_de_propiedades_psicometricas_de_un_cuestionario_de_afrontamiento_apoyo_social_y_conducta_alimentaria_en_personas_ con_diabetes_e_hipertension

14. Acrich de Gutmann L. La evaluación de las redes de apoyo social: el cuestionario SSQ. [Internet]. Argentina: XIII Jornadas de Investigación y Segundo Encuentro de Investigadores en Psicología del Mercosur; 2006 [Citado 25 Enero 2018]. 2 p. Disponible en: https://www.aacademica.org/000039/373.pdf

15. Sherbourne, CD, Stewart AL. The MOS Social Support Survey. Soc. Sci. Med [Internet]. 1991 [Citado 26 Enero 2018];32(6):705-4. Disponible en: https://www.ncbi.nlm.nih. gov/pubmed/2035047

16. Polit PD, Beck Tatano B. Investigación en enfermería. Fundamentos para el uso de la evidencia en la práctica de la enfermería. 9na ed. Filadelfia: Lippincott Williams \& Wilkins; c2018. 442 p.

17. González-Ramírez MT, Landero Hernández R, Díaz Rodríguez C. Propiedades Psicométricas de una Escala para Evaluar el Apoyo Social a través de las Redes Sociales. Acta de Investigación Psicológica [Internet]. 2013 [Citado 28 Enero 2018];3(3):1238-47. Disponible en: https://www.sciencedirect.com/science/article/pii/S2007471913709633

18. REGLAMENTO de la Ley General de Salud en Materia de Investigación para la Salud. Diario Oficial de la Federación [nternet].1987 [Citado 20 Marzo 2018]; Disponible en: http:// www.salud.gob.mx/unidades/cdi/nom/compi/rlgsmis.html

19. Declaración de Helsinki de la AMM. Principios éticos para las investigaciones médicas en seres humanos. Asociación Médica Mundial [Internet]. 2013 [Citado 20 Marzo 2018]; Disponible en: https://www.wma.net/es/policies-post/declaracion-de-helsinki-de-la-amm-principios-eticos-para-las-investigaciones-medicas-en-seres-humanos/

20. Schütz Balistieri A, Mara de MeloTavares C. La importancia del apoyo socio-emocional en adolescentes y adultos jóvenes portadores de enfermedad crónica: una revisión de literatura. Enferm. glob. [Internet]. 2013 [Citado 11 Junio 2018];12(30):. Disponible en: http://scielo.isciii.es/scielo. php? script=sci_arttext\&pid=S1695-61412013000200019

21. Martínez, RS. Redes virtuales y apoyo social percibido en usuarios con discapacidad: análisis según la tipología, grado y etapa en la que se adquiere la discapacidad. Escritos de Psicología [Internet]. 2017 [Citado 12 Junio 2018];10(1):31-40. Disponible en: http://www.redalyc.org/ pdf/2710/271051044003.pdf

22. García-Jiménez A, López de Ayala MC, Catalina García B. Hábitos de uso en Internet y en las redes sociales de los adolescentes españoles. Revista Científica de Comunicación y Educación [Internet]. 2013 [Citado 13 Junio 2018];21(41):195204. Disponible en: https://www.revistacomunicar.com/index. php? contenido $=$ detalles $\&$ numero $=41 \&$ articulo $=41-2013-19$ 